\title{
Theory of differential rotation and meridional circulation
}

\author{
Leonid L. Kitchatinov \\ Institute for Solar-Terrestrial Physics, \\ Lermontov Str. 126a, PO Box 291, Irkutsk 664033, Russia \\ email: kit@iszf.irk.ru \\ Pulkovo Astronomical Observatory, \\ St. Petersburg 196140, Russia
}

\begin{abstract}
Meridional flow results from slight deviations from the thermal wind balance. The deviations are relatively large in the boundary layers near the top and bottom of the convection zone. Accordingly, the meridional flow attains its largest velocities at the boundaries and decreases inside the convection zone. The thickness of the boundary layers, where meridional flow is concentrated, decreases with rotation rate, so that an advection-dominated regime of dynamos is not probable in rapidly rotating stars. Angular momentum transport by convection and by the meridional flow produce differential rotation. The convective fluxes of angular momentum point radially inward in the case of slow rotation but change their direction to equatorward and parallel to the rotation axis as the rotation rate increases. The differential rotation of main-sequence dwarfs is predicted to vary mildly with rotation rate but increase strongly with stellar surface temperature. The significance of differential rotation for dynamos has the opposite tendency to increase with spectral type.
\end{abstract}

Keywords. Sun: rotation, stars: rotation, stars: activity, hydrodynamics

\section{Introduction}

Differential rotation and meridional flow are the two main components of global stellar circulation. Both are important for dynamos. Differential rotation winds toroidal magnetic fields, and meridional flow near the base of the convection zone is probably responsible for the observed equatorward migration of sunspot activity. Knowledge of differential rotation as a function of stellar parameters is a key for understanding stellar dynamos.

Helioseismology has shown that the regions inside the sun occupied by thermal convection and differential rotation coincide. The same is probably true of the meridional flow. Any theory of differential rotation and meridional flow has to describe the global flows against the background of convective turbulence. The large-scale and turbulent flows are intimately linked. The theory, therefore, has to rely on the tools and methods of the mean-field hydrodynamics of turbulent fluids.

This paper reviews the mean-field theory of meridional flow and differential rotation. The differential rotation can be understood as a result of interaction between convection and rotation. The meridional flow is produced by the non-conservative part of centrifugal force and by the baroclinic torque provided that these two drivers do not balance each other. Numerical models based on the theory are also discussed. The models reproduce closely solar rotation and predict the dependence of differential rotation on stellar parameters. The results of 3D numerical simulations are discussed only occasionally. Numerical experiments have been reviewed recently by Miesch \& Toomre (2009) and Brun \& Rempel (2009). 


\section{Meridional circulation}

Meridional circulation is a vortical flow. Accordingly, the flow is convenient to describe in terms of the azimuthal vorticity $\omega=\left(\boldsymbol{\nabla} \times \boldsymbol{V}^{\mathrm{m}}\right)_{\phi}$, where $\boldsymbol{V}^{\mathrm{m}}$ is the global (azimuthallyaveraged) meridional velocity. The mean-field vorticity equation,

$$
\frac{\partial \omega}{\partial t}+r \sin \theta \boldsymbol{\nabla} \cdot\left(\boldsymbol{V}^{\mathrm{m}} \frac{\omega}{r \sin \theta}\right)+\mathcal{D}\left(\boldsymbol{V}^{\mathrm{m}}\right)=\sin \theta r \frac{\partial \Omega^{2}}{\partial z}-\frac{g}{c_{\mathrm{p}} r} \frac{\partial S}{\partial \theta},
$$

collects the meridional flow drivers on its right side while the left side describes the flow reaction to this driving. In particular, the term $\mathcal{D}\left(\boldsymbol{V}^{\mathrm{m}}\right)$ accounts for the viscous resistance by the eddy viscosity to the meridional flow driving,

$$
\mathcal{D}\left(\boldsymbol{V}^{\mathrm{m}}\right)=-\varepsilon_{\phi j k} \frac{\partial}{\partial r_{j}}\left(\frac{1}{\rho} \frac{\partial}{\partial r_{l}}\left(\rho \mathcal{N}_{k l i n} \frac{\partial V_{i}^{\mathrm{m}}}{\partial r_{n}}\right)\right),
$$

where $\mathcal{N}_{k l i n}$ is the eddy viscosity tensor and repetition of subscripts signifies summation. In Eq. (2.1), the usual spherical coordinates $(r, \theta, \phi)$ are used, $\Omega$ is the angular velocity, $S$ is the specific entropy, $g$ is gravity, $c_{\mathrm{p}}$ is the specific heat at constant pressure and $\partial / \partial z=\cos \theta \partial / \partial r-r^{-1} \sin \theta \partial / \partial \theta$ is the spatial derivative along the rotation axis.

In hydrodynamics, there are only two main sources of meridional flow illustrated by Fig. 1. The first term on the right side of Eq. (2.1) shows that differential rotation can produce the flow (Kippenhahn 1963). If angular velocity varies with the cylindrical coordinate $z$ along the rotation axis, the centrifugal force is not conservative and generates vorticity. This effect was recently named 'gyroscopic pumping' (Garaud \& Bodenheimer 2010). If angular velocity decreases with distance from the equatorial plane, as it does in the sun, the centrifugal force produces a torque driving anti-clockwise circulation (in the north-west quadrant of the meridional cross-section).
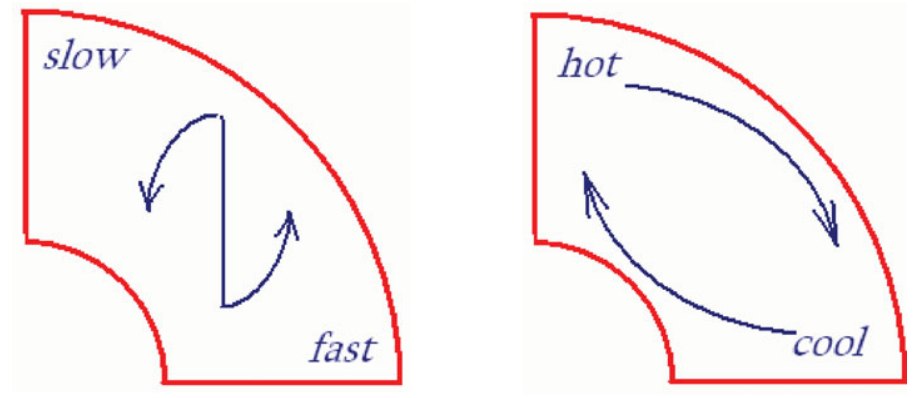

Figure 1. Illustration of centrifugal (left) and baroclinic (right) driving of the meridional flow (see text).

The second term on the right side of Eq. (2.1) allows for the baroclinic driving of the meridional flow. If polar regions are warmer than the equator, as they probably are on the sun (Rast et al. 2008), then the warm fluid tends to rise up near poles and spread over the surface while the cooler equatorial fluid tends to sink down and spread over near the base of the convection zone thus producing clockwise circulation (Fig. 1).

The two sources of the meridional flow are competing on the sun. This is probably not by chance. It seems to be a general rule that these two sources are nearly balancing each other in convective stars. If Eq. (2.1) is normalized to dimensionless units by multiplying this equation by the square of the viscous diffusion time, $R^{4} / \nu_{\mathrm{T}}^{2}$, the first term on the right side gets a coefficient of the Taylor number, Ta $=4 \Omega^{2} R^{4} / \nu_{\mathrm{T}}^{2}$; $R$ is the stellar radius and $\nu_{\mathrm{T}}$ is the eddy viscosity. This number is large on the sun, 
$\mathrm{Ta} \sim 10^{7}$. The second (baroclinic) term also gets a big coefficient of the Grashof number, $\operatorname{Gr}=\left(g R^{3} / \nu_{\mathrm{T}}^{2}\right)(\delta T / T) \sim 10^{7} \delta T_{\text {surf }} ; \delta T$ is the pole-equator temperature difference and $\delta T_{\text {surf }}$ is the surface value of this differential temperature. The left side of the equation, on the contrary, scales with the much smaller Reynolds number $\operatorname{Re}=V_{0} R / \nu_{\mathrm{T}} \sim 10$, where $V_{0} \sim 10 \mathrm{~m} / \mathrm{s}$ is the characteristic amplitude of the meridional flow (the second term on the left side of (2.1) scales with $\operatorname{Re}^{2}$ ). Therefore, the Eq. (2.1) has to be satisfied by the two terms on the right side nearly balancing each other.

Neglecting the left side in Eq. (2.1) gives the famous equation of thermal wind balance

$$
0=\sin \theta r \frac{\partial \Omega^{2}}{\partial z}-\frac{g}{c_{\mathrm{p}} r} \frac{\partial S}{\partial \theta} .
$$

The equation shows in particular that a positive differential temperature of the order of $1 \mathrm{~K}$ is necessary for isorotational surfaces to deviate considerably from cylinders in solar rotation models.

Equation (2.3) shows what the thermal wind balance is but it does not explain how the balance is maintained. In order to understand this, we have to return to the meridional flow equation (2.1) and imagine that the balance is somehow violated. This gives a strong source of meridional flow. The excited flow reacts back on the distributions of angular velocity and entropy by transporting angular momentum and heat to reestablish the balance. Meridional flow maintains the thermal wind balance and deviations from the balance drive the flow.
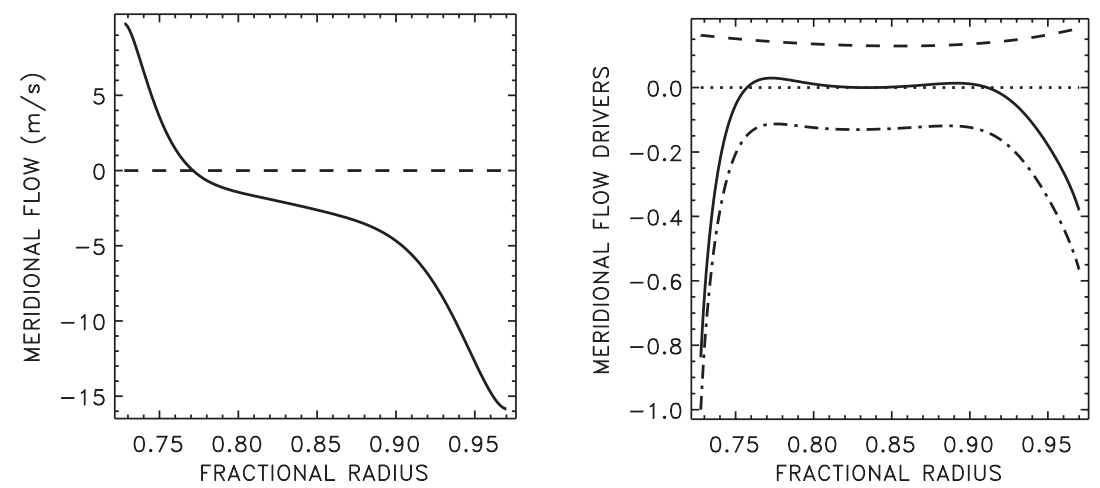

Figure 2. Left: depth profile of the meridional velocity for $45^{\circ}$ latitude after the mean-field model of Kitchatinov \& Olemskoy (2011). Negative velocity means poleward flow. Right: dependencies of the baroclinic (dashed) and centrifugal (dashed-dotted) terms of Eq. (2.1) and their sum (full line) on depth for the same latitude. The meridional flow attains its largest velocities in the boundary layers where the thermal wind balance is violated.

This qualitative picture can explain why the meridional flow decreases with depth beneath the solar surface (Zhao \& Kosovichev 2004). It does so because the stress-free conditions on the boundaries of the convection zone are not compatible with thermal wind balance. Accordingly, the boundary layers form where the balance is violated (Durney 1989). As deviations from the balance produce the meridional flow, the meridional velocity attains its maximum values at the top and bottom boundaries and decreases inside the convection zone. Fig. 2 illustrates the thermal wind balance and the meridional flow structure typical of mean-field models of global solar circulation. Boundary layers were found also in the 3D simulations of Brown et al. (2008). The bottom flow of Fig. 2 is not small compared to the surface flow. The flow, however, decreases rapidly with depth beneath the base of the convection zone (Gilman \& Miesch 2004). 
Fig. 2 shows the boundary layers where the thermal wind balance is violated. The bulk of the convection zone is, nevertheless, very close to the balance. Even there, however, the balance condition is satisfied only on average (Fig. 2 results from the mean-field model). Differential rotation is produced by turbulent convection, so that fluctuating deviations from the thermal wind balance are unavoidable (Brun et al. 2010). As the deviations produce the meridional flow, the flow is expected to fluctuate considerably with time. This qualitative picture was reproduced quantitatively by Rempel (2005) whose global circulation model allowed for random fluctuations in the $\Lambda$-effect (cf. the next Section for the $\Lambda$-effect definition). The fluctuating $\Lambda$-effect produces small fluctuations in differential rotation, which in turn produce much larger irregular variations in the meridional flow. The relative amplitude of fluctuations of the meridional flow in Rempel's model was about two orders of magnitude larger compared to fluctuations in angular velocity. Helioseismologically detected meridional flow indeed shows considerable changes from year to year (Zhao \& Kosovichev 2004; Gonzáles Hernández et al. 2006) contrasting the slight variations in the rotation rate.

\section{Differential rotation}

Helioseismology shows that differential rotation and thermal convection occupy the same region inside the sun. The entire convection zone is rotating differentially while the rotation inhomogeneity decreases rapidly with depth beneath the convection zone (Wilson et al. 1997; Schou et al. 1998). This supports the theoretical concept pioneered by Lebedinskii (1941) that the differential rotation results from interaction between convection and rotation. Convection in a rotating fluid is disturbed by the Coriolis force. The back reaction disturbs rotation to make it non-uniform.

The mean-field equation for angular velocity,

$$
\rho r^{2} \sin ^{2} \theta \frac{\partial \Omega}{\partial t}=-\nabla \cdot\left(\rho r \sin \theta\left\langle u_{\phi} \boldsymbol{u}\right\rangle+\rho r^{2} \sin ^{2} \theta \Omega \boldsymbol{V}^{\mathrm{m}}\right),
$$

shows that the angular velocity distribution can be modified by angular momentum transport, by convection $(\boldsymbol{u})$ and by meridional flow $\left(\boldsymbol{V}^{\mathrm{m}}\right)$.

\subsection{The $\Lambda$-effect}

The ability of convective motions to transport angular momentum even in the case of uniform rotation is called the ' $\Lambda$-effect' (Rüdiger 1989). The uniformity of rotation is mentioned because the eddy viscosity can transport angular momentum also if rotation is not homogeneous. The viscosity, however, tends to diminish the differential rotation, not to produce it, in contrast with the non-diffusive $\Lambda$-effect.

Eq. (3.1) shows that the azimuthal and meridional convective velocities have to be correlated for the $\Lambda$-effect to emerge. The finite cross-correlation requires the convective turbulence to possess a preferred direction of anisotropy or inhomogeneity (cf. Kitchatinov (2011) for pictorial discussion of the origin of the $\Lambda$-effect). The radial preferred direction is imposed by gravity. The $\Lambda$-effect is allowed for by the non-diffusive part $Q_{i j}^{\Lambda}$ of the velocity correlation tensor $Q_{i j}=\left\langle u_{i} u_{j}\right\rangle$. The radial $\left(Q_{r \phi}^{\Lambda}\right)$ and meridional $\left(Q_{\theta \phi}^{\Lambda}\right)$ non-diffusive fluxes of angular momentum for radially-stratified fluid read

$$
\begin{aligned}
Q_{r \phi}^{\Lambda} & =-\nu_{\mathrm{T}}\left(\frac{\ell}{H_{\rho}}\right)^{2} \Omega \sin \theta\left(V\left(\Omega^{*}\right)+H\left(\Omega^{*}\right) \cos ^{2} \theta\right), \\
Q_{\theta \phi}^{\Lambda} & =\nu_{\mathrm{T}}\left(\frac{\ell}{H_{\rho}}\right)^{2} \Omega \sin ^{2} \theta \cos \theta H\left(\Omega^{*}\right),
\end{aligned}
$$


where $\ell$ is the correlation length,

$$
\nu_{\mathrm{T}}=-\frac{\tau \ell^{2} g}{15 c_{\mathrm{p}}} \frac{\partial S}{\partial r}
$$

is the background eddy viscosity, and $\tau$ is the convective turnover time. The dimensionless functions $V\left(\Omega^{*}\right)$ and $H\left(\Omega^{*}\right)$ in Eq. (3.2) depend on the Coriolis number

$$
\Omega^{*}=2 \tau \Omega
$$

which is the key parameter of the differential rotation theory. The parameter measures intensity of interaction between convection and rotation. Its value defines whether the convective eddies are living long enough for rotation to influence them considerably. The Coriolis number (3.4) depends on depth in the convection zone. It is smaller than one near the solar surface but increases with depth to exceed ten near the base of the convection zone. Therefore, the $\Lambda$-effect theory should be nonlinear in $\Omega^{*}$.
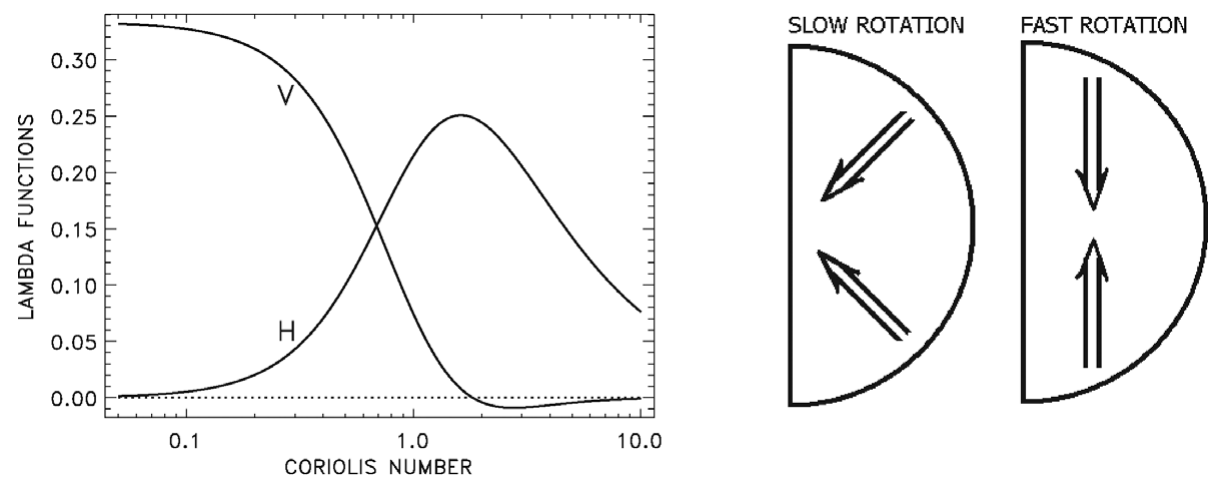

Figure 3. The functions $V\left(\Omega^{*}\right)$ and $H\left(\Omega^{*}\right)$ of the $\Lambda$-effect of Eq. (3.2) derived by Kitchatinov \& Rüdiger $(1993,2005)$. The right panel shows the directions of angular momentum transport for the cases of slow $\left(\Omega^{*} \ll 1\right)$ and fast $\left(\Omega^{*} \gg 1\right)$ rotation.

The function $V\left(\Omega^{*}\right)$ in Eq. (3.2) is the normalized vertical flux of angular momentum. Positive $V$ means radial inward transport. The function $H\left(\Omega^{*}\right)$ is the normalized flux parallel to the rotation axis. Its positive value means that the angular momentum is transported towards the equatorial plane. The two (not mutually orthogonal) components of the angular momentum fluxes are shown in Fig. 3 as functions of the Coriolis number. In the case of slow rotation, $\Omega^{*} \ll 1$, the $H$-function is relatively small and angular momentum is transported downward in radius. In the opposite case of rapid rotation, $\Omega^{*} \gg 1$, the $V$-function is small, and the angular momentum flux is parallel to the rotation axis pointing to the equatorial plane. As the Coriolis number increases from small to large values, the direction of the non-diffusive flux of angular momentum varies smoothly from radial inward to equatorward and parallel to the rotation axis.

\subsection{Eddy viscosities}

After the significance of the correlation of azimuthal and meridional convective velocities for differential rotation was recognized, there were attempts to observe this correlation using sunspots as tracers (Ward 1965). The first measurements gave finite correlation, $Q_{\theta \phi} \cos \theta \sim 10^{3} \mathrm{~m}^{2} / \mathrm{s}^{2}$, with positive $Q_{\theta \phi}$ in the northern and negative in the southern hemisphere, indicating the equatorward transport of angular momentum. Subsequent measurements of Ribes (1986) and Nesme-Ribes et al. (1993), however, did not confirm this result. 
The equatorial acceleration does not demand a certain sign of $Q_{\theta \phi}$ (a positive value of $Q_{\theta \phi} \cos \theta$ would mean that angular momentum is permanently pumped towards the equator implying an increase of differential rotation with time). The total correlation,

$$
Q_{i j}=Q_{i j}^{\Lambda}+Q_{i j}^{\nu},
$$

includes the contribution $Q^{\nu}$ of the eddy viscosities in line with the $\Lambda$-effect. The viscous fluxes of angular momentum,

$$
\begin{aligned}
Q_{r \phi}^{\nu} & =-\nu_{1} r \sin \theta \frac{\partial \Omega}{\partial r}-\nu_{2} \sin \theta \cos \theta\left(r \cos \theta \frac{\partial \Omega}{\partial r}-\sin \theta \frac{\partial \Omega}{\partial \theta}\right) \\
Q_{\theta \phi}^{\nu} & =-\nu_{1} \sin \theta \frac{\partial \Omega}{\partial \theta}+\nu_{2} \sin ^{2} \theta\left(r \cos \theta \frac{\partial \Omega}{\partial r}-\sin \theta \frac{\partial \Omega}{\partial \theta}\right)
\end{aligned}
$$

(Kitchatinov et al. 1994), include two viscosity coefficients, $\nu_{1}=\nu_{\mathrm{T}} f_{1}\left(\Omega^{*}\right)$ and $\nu_{2}=$ $\nu_{\mathrm{T}} f_{2}\left(\Omega^{*}\right)$. Rotating convection is anisotropic and the eddy viscosity $\nu_{1}$ acting in the direction normal to the rotation axis differs from the viscosity $\nu_{1}+\nu_{2}$ for the direction parallel to the axis.

A positive non-diffusive part, $Q_{\phi \theta}^{\Lambda} \cos \theta>0$, is required for maintaining equatorial acceleration. It is hardly possible, however, to separate the contributions of the $\Lambda$-effect from the contribution of the eddy viscosities in the total correlation (3.5) supplied by observations. The same separation problem is met by $3 \mathrm{D}$ numerical simulations of the $\Lambda$-effect. It was, nevertheless, possible to extract the $Q^{\Lambda}$ from the numerical simulations by Käpylä \& Brandenburg (2008) and Käpylä et al. (2011). The analytical results in Fig. 3 generally agree with the simulations of the $\Lambda$-effect.

\subsection{Surface shear layer}

The natural boundary condition for the differential rotation problem is the requirement that the surface density of external forces be zero, $Q_{r \phi}=Q_{r \theta}=0$. The stress-free condition means that the global flows are produced by internal processes in the convection zone, not imposed externally. The condition on the upper boundary is simplified by the small value of the Coriolis number so that the angular momentum fluxes (3.2) and (3.6) can be taken in the limit of $\Omega^{*} \rightarrow 0$. In this limit, the viscosity $\nu_{2}$ in Eq. (3.6) vanishes and $\nu_{1}=\nu_{\mathrm{T}}$. The top boundary condition, $Q_{r \phi}=0$, then reads

$$
r \frac{\partial \Omega}{\partial r}=-\left(\frac{\ell}{H_{\rho}}\right)^{2} V(0) \Omega \text { at } r=R .
$$

With positive $V(0)$ of Fig. 3, Eq. (3.7) requires the angular velocity to increase with depth near the surface in at least qualitative agreement with the surface shear layer detected by helioseismology (Schou et al. 1998).

We have seen in Sect. 2 that the meridional flow also has a near-surface boundary layer. The near-surface rotational shear and meridional flow are mutually related. This relation was recently analyzed by Miesch \& Hindman (2011).

\section{Differential temperature}

It is not possible to reproduce the helioseismological rotation law by differential rotation models if the differential temperature is not allowed for (Brandenburg et al. 1990; Rüdiger et al. 2005; Miesch et al. 2006).

Similar to the differential rotation, the differential temperature is currently understood as a result of rotational influence on convection. The tensor $\chi_{i j}$ of the eddy thermal 
diffusion that controls the convective heat flux,

$$
F_{i}^{\mathrm{conv}}=-\rho T \chi_{i j} \frac{\partial S}{\partial r_{j}}
$$

includes the rotationally-induced anisotropy and quenching

$$
\chi_{i j}=\chi_{\mathrm{T}}\left(\phi\left(\Omega^{*}\right) \delta_{i j}+\phi_{\|}\left(\Omega^{*}\right) \frac{\Omega_{i} \Omega_{j}}{\Omega^{2}}\right), \quad \chi_{\mathrm{T}}=-\frac{\tau \ell^{2} g}{12 c_{\mathrm{p}}} \frac{\partial S}{\partial r} .
$$

The diffusivity quenching functions of the Coriolis number (3.4) are shown in Fig. 4.

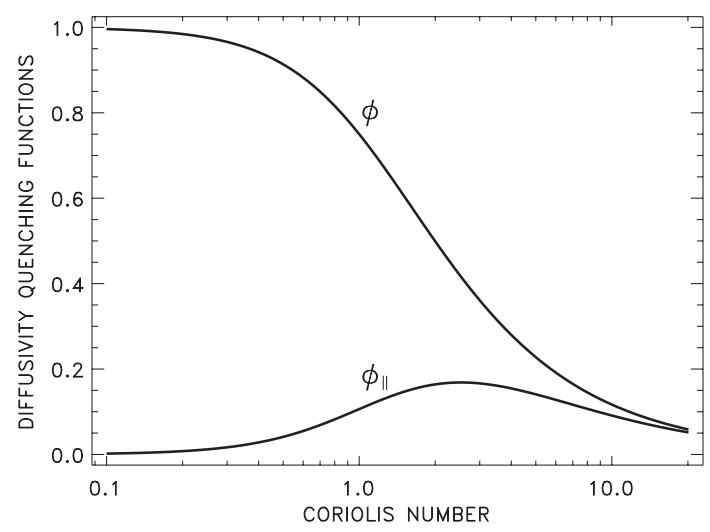

Figure 4. Quenching functions of the eddy thermal diffusivity of Eq. (4.2) after the quasilinear theory of turbulent transport in rotating fluids (Kitchatinov et al. 1994).

Directions of the entropy gradient and heat flux (4.1) do not coincide due to the anisotropy of heat transport. Even if entropy varies mainly with radius, the meridional flux,

$$
F_{\theta}^{\text {conv }}=\rho T \chi_{\mathrm{T}} \phi_{\|}\left(\Omega^{*}\right) \cos \theta \sin \theta \frac{\partial S}{\partial r},
$$

does not vanish. For the negative radial gradient of entropy in convection zones, the meridional flux (4.3) transports heat to the poles. There have been multiple attempts to observe differential temperature on the sun. Recent observations of Rust et al. (2008) suggest that polar regions are warmer than the equator by about $2.5 \mathrm{~K}$. After the Eq. (2.3) of thermal wind balance, this small differential temperature suffices for the isorotational surfaces to deviate considerably from cylinders.

\section{Differential rotation models}

What is usually meant by the 'differential rotation models' are numerical solvers which define not only the angular velocity but also meridional flow and entropy distributions in stellar convection zones.

\subsection{The Sun}

The solar rotation model in Fig. 5 is close to the helioseismological rotation law. This Figure was computed by using two different models - one for the convection zone, and another model for the tachocline and radiation zone rotation. Physical conditions in convection and radiation zones differ so much that it is not possible to cover both in one model. The tachocline modeling did not influence the computation of differential rotation 
in the convection zone in any way but just used the results of this computation as the top boundary condition.
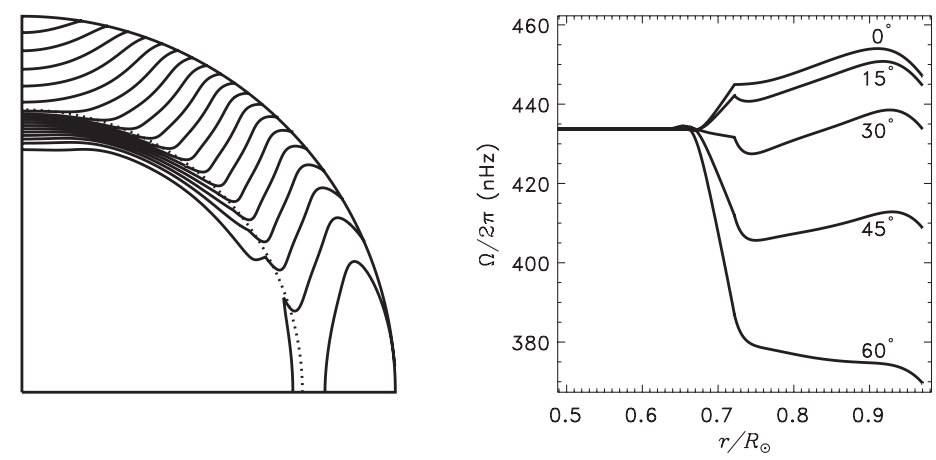

Figure 5. Angular velocity isolines (left) and depth-dependencies of rotation frequencies for several latitudes (right) after the mean-filed model of Kitchatinov \& Olemskoy (2011).

The model of the convection zone rotation in Fig. 5 was tuned with one free parameter. Mean-field models can avoid arbitrary prescriptions of any parameters. All the modeling needs, including the $\Lambda$-effect, the eddy viscosities and thermal conductivities, has been derived using the same quasi-linear approximation of the mean-field theory. These derivations, formally, leave no freedom for model design. However, the quasi-linear approximation is not expected to be very precise. This raises the question of how sensitive the model is to variations of basic parameters. The sensitivity is normally quite low, except for one parameter. E.g., if the $\Lambda$-effect is reduced by $50 \%$ by multiplying the non-diffusive fluxes of Eq. (3.2) by a factor of 0.5 , the surface differential rotation in the solar model decreases by only $10 \%$ : from $30 \%$ to $27 \%$. It is remarkable that increasing the $\Lambda$-effect by $50 \%$ (factor 1.5 ) also reduces the differential rotation by same $10 \%$. Sensitivity to an increase of eddy viscosities or heat conductivities is also rather low (decreasing the eddy diffusion can make the model unstable to thermal convection and, therefore, inconsistent). The model, however, is rather sensitive to the anisotropy of thermal conductivity. If the parameter $C_{\chi}$ is introduced in Eq. (4.2),

$$
\chi_{i j}=\chi_{\mathrm{T}}\left(\phi\left(\Omega^{*}\right) \delta_{i j}+C_{\chi} \phi_{\|}\left(\Omega^{*}\right) \frac{\Omega_{i} \Omega_{j}}{\Omega^{2}}\right),
$$

the resulting differential rotation reacts quite sensitively to variations of this parameter and generally increases with $C_{\chi}$. Fig. 5 was obtained with $C_{\chi}=1.5$. This implies that the quasi-linear theory underestimates the anisotropy of thermal eddy conductivity. All the stellar models discussed below were obtained with the same value of $C_{\chi}=1.5$.

Meridional flow for the same model as Fig. 5 is shown in Fig. 2.

\subsection{Solar twins}

Differential rotation has already been measured for many solar-type stars (Korhonen 2012) motivating stellar applications of differential rotation models. Figures 6 and 7 show the differential rotation and meridional flow computed for two stars similar to the sun in mass but rotating faster (Kitchatinov \& Olemskoy 2011).

Croll et al. (2006) measured the differential rotation of a solar twin $\epsilon$ Eridani using very precise photometry of the MOST mission. The star is younger than the sun and rotates with a shorter period of $P_{\text {rot }} \simeq 11$ days. The differential rotation and meridional flow computed for this star are shown in Fig. 6. The computations give the relative 

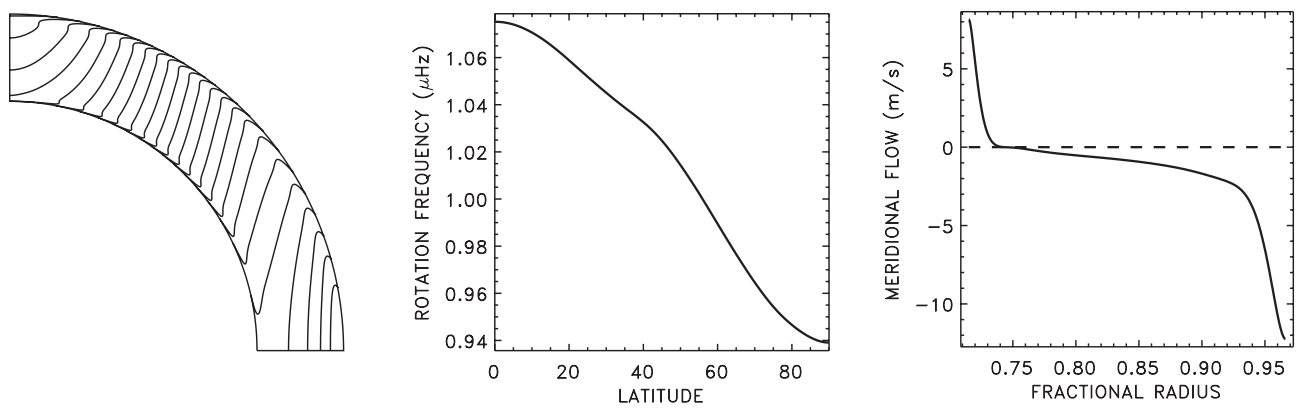

Figure 6. Differential rotation and meridional flow computed for solar-type star $\epsilon$ Eridani. From left to right: angular velocity isolines, surface profile of rotation rate, and depth profile of the meridional flow at $45^{\circ}$-latitude.

magnitude,

$$
\alpha_{\mathrm{DR}}=1-\frac{\Omega_{\mathrm{pole}}}{\Omega_{\mathrm{eq}}},
$$

of the surface differential rotation of $\epsilon$ Eri of $\alpha_{\mathrm{DR}}=0.13$ close to the value of $\alpha_{\mathrm{DR}}=0.11$ measured by Croll et al. (2006). The absolute value of the surface differential rotation is close to that of the sun.
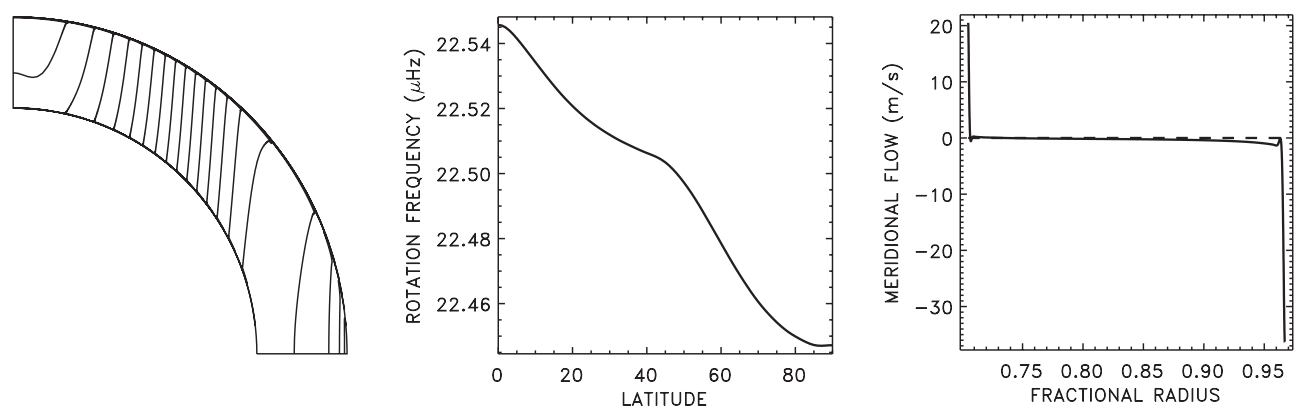

Figure 7. Differential rotation and meridional flow computed for the young rapidly rotating dwarf AB Doradus. From left to right: angular velocity isolines, surface profile of rotation rate, and depth profile of the meridional flow at $45^{\circ}$-latitude.

Fig. 7 shows differential rotation and meridional flow computed for the very rapidly rotating $\left(P_{\text {rot }}=0.51\right.$ day) young dwarf AB Doradus. The computed value of $\alpha_{\mathrm{DR}}=$ $4.4 \times 10^{-3}$ is again close to the differential rotation measured by Donati \& Collier Cameron (1997) by the method of Doppler imaging. Also in this case the differential rotation in dimensional units is close to the solar value.

The surface profiles of Figs. 6 and 7 are not as smooth as it is on the sun. There are peculiarities positioned around the latitude, where the isoline of angular velocity tangential to the inner boundary at the equator arrives on the surface.

The meridional flows of these Figures differ from the flow computed for the sun (Fig. 2) by their higher concentration at the boundaries of the convection zone. The boundary layers discussed in Sect. 2 become thinner as rotation rate increases. The flow of Fig. 7 in the very rapidly rotating star consists of two near-boundary jets linked by very slow circulation in the bulk of the convection zone. The meridional flow is believed important for the solar dynamo (Choudhuri 2008). It is, however, hard to imagine that the surface-jets flow of Fig. 7 can be significant for dynamos. As a star ages and its rotation rate decreases, the boundary layers broaden (Fig. 6) and the meridional flow eventually 
transforms to a distributed flow as in Fig. 2. It may be expected that the dynamo of a star changes to the advection-dominated type from some other dynamo regime, where meridional flow is not significant, as rotation rate decreases. This may be the reason for the presence of two separate branches for slow and fast rotators in the observed dependence of the stellar activity cycle periods on rotation rate (Saar \& Brandenburg 1999).

\subsection{Mass and rotation rate dependence}

Applications of the mean-field model to individual stars do not always agree with observations so closely as in the two examples given above. However, no one case of clear disagreement has been met so far. This motivates using the model to explore the dependence of differential rotation on stellar parameters. Fig. 8 shows the surface differential rotation computed for main-sequence stars of different mass and age. The rotation periods of this Figure were specified using Gyrochronology (Barnes 2007, 2009).
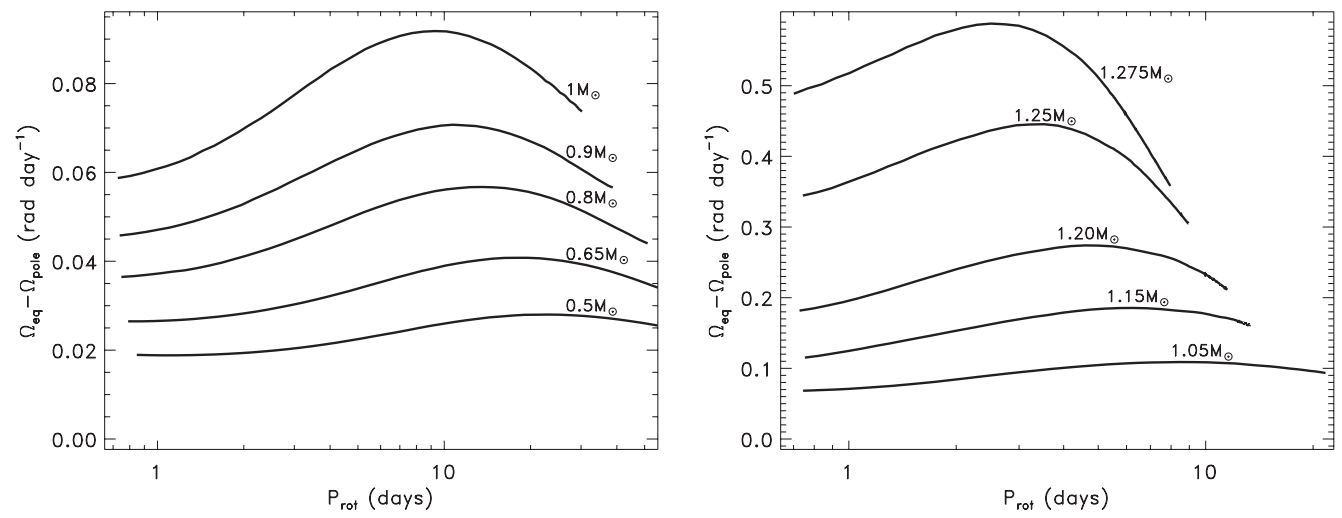

Figure 8. Surface differential rotation as a function of the rotation period for main-sequence dwarfs of subsolar (left) and supersolar (right) masses (from Kitchatinov \& Olemskoy 2012).

Fig. 8 suggests that the differential rotation of a star of given mass varies by about $30 \%$ only as the star ages. A much stronger tendency is the increase in differential rotation with stellar mass. The same tendency for rapidly rotating stars was observed by Barnes et al. (2005).

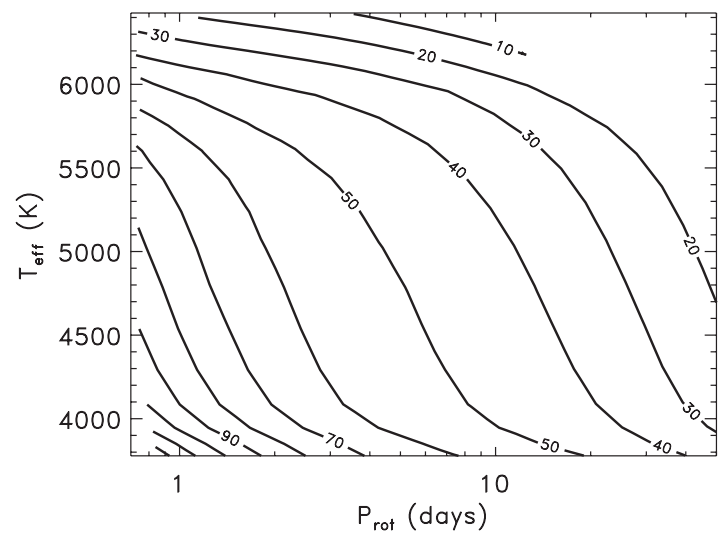

Figure 9. Isolines of the $C_{\Omega}$ dynamo number (5.3) from the same computations as Fig. 8. The dynamo number increases with rotation rate but decreases with temperature.

Differential rotation takes part in stellar dynamos by winding the toroidal field lines. 
The computations in Fig. 8 predict that the largest differential rotation belongs to the hottest F-stars in this Figure. The question arises whether the strong differential rotation implies high dynamo activity. The computations suggest a negative answer. The efficiency of differential rotation in generating magnetic fields can be estimated by the modified magnetic Reynolds number that in dynamo theory is conventionally notated as $C_{\Omega}$,

$$
C_{\Omega}=\frac{\Delta \Omega H^{2}}{\eta_{\mathrm{T}}}
$$

where $\Delta \Omega$ is the angular velocity variation within the convection zone, $H$ is the convection zone thickness, and $\eta_{\mathrm{T}}$ is the magnetic eddy diffusivity. This number estimates the ratio of the rate $\Delta \Omega$ of magnetic field production by differential rotation to the rate $H^{2} / \eta_{\mathrm{T}}$ of diffusive decay of the field. Fig. 9 shows isolines of $C_{\Omega}$ on the plane of effective temperature and rotation period, obtained in the same computations as Fig. 8. $C_{\Omega}$ increases with rotation rate. This is mainly due to the rotational quenching of the eddy diffusivity. The dynamo number (5.3) increases also with decreasing temperature. This is mainly because convection is slower and $\eta_{\mathrm{T}}$ is smaller in cooler stars. The increase of $\eta_{\mathrm{T}}$ with increasing temperature overpowers the increase in differential rotation, so that the dynamo-number (5.3) decreases.

The large rotational shear of F-stars is less efficient in winding magnetic fields than the small differential rotation of K-dwarfs. The widely spread opinion that low mass stars host $\alpha^{2}$-dynamos producing non-axisymmetric global fields is not supported by the estimations of the $C_{\Omega}$ dynamo-number. The small but dynamo-efficient differential rotation of these stars favors axisymmetric global fields. This might be the reason why the magnetic structure of the M-star observed by Donati et al. (2006) was close to axial symmetry.

\section{Summary}

We have seen that meridional flow results from (slight) deviations from the thermal wind balance. The balance, in turn, is maintained by the flow. Meridional flow attains its largest velocities in the boundary layers near the top and bottom of the convection zone where deviations from the thermal wind balance are relatively large. The thickness of these layers decreases with increasing rotation rate. The layers in rapidly rotating stars are so thin that an advection-dominated regime of the dynamo in these stars is not probable.

Differential rotation is produced mainly by convection and also by the meridional flow transporting angular momentum. Differential temperature with poles warmer than the equator is very significant for differential rotation formation. Differential temperature is currently understood as a result of rotationally induced anisotropy of convective heat transport. The anisotropy is the parameter to which differential rotation models are most sensitive.

Differential rotation models reproduce closely the helioseismological rotation law and the observed differential rotation of several individual stars. The models predict the differential rotation of a star of given mass to vary mildly as the star ages and its rotation rate decreases. Differential rotation is, however, predicted to increase strongly with stellar mass. The significance of rotational shear for dynamos estimated with the $C_{\Omega}$ dynamo number (5.3) has the opposite tendency. The large differential rotation in F-stars is less dynamo-efficient than the tiny rotation inhomogeneity of M-stars. 


\section{Acknowledgements}

The author is thankful to the Russian Foundation for Basic Research (projects 10-0200148 and 12-02-92691_Ind) and to the Ministry of Education and Science of the Russian Federation (contract 16.518.11.7065) for their support.

\section{References}

Barnes, S. A. 2007, ApJ, 669, 1167

Barnes, S. A. 2009, in: E.E. Mamajek, D. R. Soderblom \& R.F.G. Wyse (eds.), The Ages of Stars, Proc. IAU Symposium 258, p.345

Barnes, J. R., Collier Cameron, A., Donati, J.-F., James, D. J., Marsden, S. C., \& Petit, P. 2005, MNRAS, 357, L1

Brandenburg, A., Tuominen, I., Moss, D., \& Rüdiger, G. 1990, Solar Phys., 128, 243

Brun, A. S. \& Rempel, M. 2009, Space Sci. Revs, 144, 151

Brun, A. S., Antia, H. M., \& Chitre, S. M. 2010, A\&A, 510, 33

Brown, B. P., Browning, M. K., Brun, A. S., Miesch, M. S., \& Toomre, J. 2008, ApJ, 689, 1354

Choudhuri, A. R. 2008, Adv. Sp. Res., 41, 868

Croll, B., Walker, G. A. H., Kuschnig, R., et al. 2006, ApJ, 648, 607

Donati, J.-F. \& Collier Cameron, A. 1997, MNRAS, 291, 1

Donati, J.-F., Forveille, T., Collier Cameron, A., Barnes, J. R., Delfosse, X., Jardine, M. M., \& Valenti, J. A. 2006, Science, 311, 633

Durney, B. R. 1989, ApJ, 338, 509

Garaud, P. \& Bodenheimer, P. 2010, ApJ, 719, 313

Gilman, P. A. \& Miesch, M. S. 2004, ApJ, 611, 568

Gonzáles Hernández, I., Komm, R., Hill, F., \& Howe, R. 2006, ApJ, 638, 576

Käpylä, P. J. \& Brandenburg, A. 2008, A\& A, 488, 9

Käpylä, P. J., Mantere, M. J., Guerrero, G., Brandenburg, A., \& Chatterjee, P. 2011, A\& A, 531, A162

Kippenhahn, R. 1963, ApJ, 137, 664

Kitchatinov, L. L. 2011, ASInC, 2, 71

Kitchatinov, L. L. \& Olemskoy, S. V. 2011, MNRAS, 411, 1059

Kitchatinov, L. L. \& Olemskoy, S. V. 2012, MNRAS, 423, 3344

Kitchatinov, L. L. \& Rüdiger, G. 1993, $A \mathscr{E} A$, 276, 96

Kitchatinov, L. L. \& Rüdiger, G. 2005, AN, 326, 379

Kitchatinov, L. L., Pipin, V. V., \& Rüdiger, G. 1994, AN, 315, 157

Korhonen, H. 2012, in: C. H. Mandrini \& D.F. Webb (eds.), Comparative Magnetic Minima: Characterizing quiet times in the Sun and stars, Proc. IAU Symposium 286, p.268

Lebedinskii, A. I. 1941, Astron. Zh., 18, 10

Miesch, M. S. \& Hindman, B. W. 2011, ApJ, 743, 79

Miesch, M. S. \& Toomre, J. 2009, AnRFM, 41, 317

Miesch, M. S., Brun, A. S., \& Toomre, J. 2006, ApJ, 641, 618

Nesme-Ribes, E., Ferreira, E. N., \& Vince, L. 1993, A\&AA, 276, 211

Rast, M. P., Ortiz, A., \& Meisner, R. W. 2008, ApJ, 673, 1209

Ribes, E. 1986, Adv. Sp. Res., 6, 221

Rüdiger, G. 1989, Differential Rotation and Stellar Convection. Gordon \& Breach, New York

Rüdiger, G., Egorov, P., Kitchatinov, L. L., \& Küker, M. 2005, A\&\&A, 431, 345

Rempel, M. 2005, ApJ, 631, 1286

Saar, S. H. \& Brandenburg, A. 1999, ApJ, 524, 295

Schou, J., Antia, H. M., Basu, S., et al. 1998, ApJ, 505, 390

Ward, F. 1965, ApJ, 141, 534

Wilson, P. R., Burtonclay, D., \& Li, Y. 1997, ApJ, 489, 395

Zhao, J. \& Kosovichev, A. G. 2004, ApJ, 603, 776 